Thorax, 1978, 33, 137-139

\title{
Editorial
}

\section{High-density lipoprotein, low-density lipoprotein, and coronary heart disease}

Developments in coronary heart disease (CHD) epidemiology have within the past three years brought to a conclusion our longstanding preoccupation with hypercholesterolaemia. An improved understanding of lipoprotein metabolism has rendered obsolescent for most purposes the measurement of plasma total cholesterol concentration, and attention has come to rest on the distribution of cholesterol between the major lipoprotein classes. New evidence, if substantiated, will call for a major revision of our concept of the pathogenesis of coronary atherosclerosis, and the lipid infiltration theory of Virchow (1856) might prove to have been an oversimplification of the true state of affairs.

From the outset, lipoprotein analysis revealed not one but two major abnormalities in CHD. Plasma low-density lipoprotein (LDL) concentration was increased (hence hypercholesterolaemia) and high-density lipoprotein (HDL) concentration was reduced. Furthermore, the concentrations of HDL and the remainder of the plasma lipoproteins (that is, LDL and the very-low-density lipoproteins (VLDL) combined) were inversely correlated (Gofman et al., 1966). Advocacy at that time of the lipid infiltration theory of atherosclerosis and the dietary theory of hypercholesterolaemia, in the absence of any proper understanding of lipoprotein metabolism, led to the belief that the LDL abnormality was likely to be the more important. Changes in HDL were viewed as of little consequence for CHD. An authoritative statement in those earlier years (Gofman et al., 1956) that measurement of plasma lipoproteins afforded no advantage over total cholesterol concentration in the recognition of coronary risk served inadvertently only to detract further from the HDL abnormality.

Opinion remained essentially unchanged until 1975 when a differing interpretation of the evidence was reached by Miller and Miller (1975). They proposed, in agreement with Glomset (1970), that HDL was involved in normal tissue cholesterol clearance and went on to suggest that this lipoprotein played an important role in CHD. They hypothesised that a low HDL cholesterol con- centration heralded clinical coronary disease owing to its association with impaired clearance of cholesterol from the arterial wall. This same mechanism would explain the inverse association between tissue cholesterol-pool size and plasma HDL cholesterol concentration, and would account for the reduction in HDL cholesterol concentration in those who have a forboding coronary risk-factor status. In general, HDL concentrations are lower in adult males than in females, and are reduced in the presence of obesity, hypertriglyceridaemia, and glucose intolerance. High levels of habitual physical activity are associated with an increase in HDL concentration.

Perhaps the most persuasive evidence to emerge in response to the HDL hypothesis has been the findings of two prospective surveys, one in Tromso, Norway (Miller et al., 1977) and the other from Framingham (Gordon et al., 1977). In Tromso a two-year observation of 6595 men aged 20-49 years at entry disclosed that CHD-risk was inversely correlated with HDL cholesterol concentration and directly correlated with the combined concentration of cholesterol in LDL and VLDL. These relationships were independent of each other and of plasma triglyceride concentration, blood pressure, cigarette consumption, and relative body weight. The contribution of HDL cholesterol to prediction of coronary risk was threefold greater than that of cholesterol in LDL and VLDL. Using HDL and LDL cholesterol concentrations, CHD cases could be distinguished from controls with $85 \%$ success. In Framingham similar data were collected in 1025 men and 1445 women without evidence of CHD at entry between 49 and 82 years of age. In both sexes HDL cholesterol concentration was the major lipid/lipoprotein risk-factor, irrespective of the type of CHD entered into the analysis. The strength of this association was unaffected when allowance was made for other recognised risk-factors. Plasma LDL cholesterol concentration had a weaker predictive power, and total cholesterol concentration none at all.

The abnormal lipoprotein pattern in coronary atherosclerosis suggests an imbalance of lipoprotein 
metabolism within the coronary vessels, and the link between LDL and HDL might be the intimal smooth muscle cell and fibroblast. With regard to LDL, Brown and Goldstein (1976) have described how fibroblasts acquire cholesterol by binding plasma LDL to high-affinity receptors on the cell membrane surface. Thereafter, LDL is taken into the cell and the cholesterol is released for metabolic requirements. Surface receptor activity is a function of metabolic demand for cholesterol, and repletion leads to a reduction in LDL receptor synthesis through feedback inhibition. Persistently reduced LDL receptor activity, as in familial hypercholesterolaemia, is believed to lead eventually to an increase in plasma LDL concentration and coronary atheroma.

Clearance of cholesterol from the intimal cells appears to require HDL (Bondjers et al., 1977). This lipoprotein also attaches to the cell surface, but the rate of binding shows no high-affinity characteristics. Rather, HDL binding tends to a linear function of HDL concentration in the extracellular fluid (ECF). The evidence is, therefore, that while cholesterol uptake is regulated by the cell in accordance with metabolic requirements, cholesterol clearance is dependent upon the availability of a high-density lipoprotein in ECF. It is within this context, rather than in terms of hypercholesterolaemia, that we must seek the explanation for CHD.

No specific lipoprotein abnormality has so far been recognised as invariable in CHD, although such might be the case if only we were able to examine the lipoprotein spectrum in sufficient detail. In the meantime, the reduction in HDL cholesterol concentration has emerged as the most consistent of the known lipoprotein abnormalities. As a working hypothesis (and at present nothing more), the HDL abnormality in CHD could justifiably be regarded as the most common and frequently the primary defect in lipoprotein metabolism. At least this approach opens promising avenues for research at a time when CHD preventive measures based on hypercholesterolaemia do not seem to have had much success. Speculatively, a suboptimal HDL concentration in the ECF might lead to defective clearance and cell saturation with cholesterol. In response, LDL surface receptor synthesis would be suppressed and, in a manner similar to that thought to occur in familial hypercholesterolaemia, this would ultimately lead to an increase in plasma LDL concentration. Here then is another possible explanation for the hypercholesterolaemia in CHD, which has its origin in defective tissue cholesterol clearance. Some individuals might possess both genetic and acquired defects, which promote tissue and plasma cholesterol accumulation, and interaction between such characteristics is a possibility. From an epidemiological standpoint, the independent predictive power of HDL for coronary-risk might be an index of cholesterol clearance, and the residual predictive power of LDL might be due to the presence of other factors, such as a genetic defect in LDL-receptor activity, which leads to LDL accumulation. Our major challenge is the elucidation of those processes which reduce plasma HDL concentration, and here also the likelihood of interplay between genetic and acquired characteristics must be considered.

The association between plasma VLDL concentration and CHD is more pronounced in some communities than in others (for example, parts of Scandinavia as compared with North America). Some of this association, but possibly not all, appears to be due to the strong inverse correlation between plasma HDL and VLDL concentrations. A link between VLDL and HDL metabolism, as yet not fully understood, might have an important bearing on CHD. There are recent hints also of a link between chylomicron and HDL metabolism.

This new evidence in no way negates the possible importance of diet for CHD, although it does demand an urgent look at the relationship between dietary factors and HDL cholesterol. The effects, if any, of dietary fibre, trace elements, and saturated and polyunsaturated fats on HDL are largely unknown, but a high carbohydrate diet appears to reduce HDL cholesterol concentration at least in the short term. The associations of HDL with adiposity and habitual physical activity suggest that total energy balance might be important in this context.

These developments raise several intriguing possibilities, which can only be touched on here. Does the ability of moderate alcohol intake to increase plasma HDL cholesterol concentration (Castelli et al., 1977) explain the strong negative association between alcohol consumption and coronary-risk (Yano et al., 1977)? Does the sometimes dramatic fall in HDL cholesterol concentration induced by pyrexial illness tip the balance in some individuals who are at the time on the verge of a 'heart attack', thereby accounting for the reported excess of CHD seen in clinical practice during an epidemic of virus illness (Nicholls and Thomas, 1977)? Will measurement of subfractions of HDL and, in particular, the relatively cholesterol-rich fraction, $\mathrm{HDL}_{2}$, improve still $\stackrel{\mathbb{Q}}{\AA}$ further our ability to recognise coronary-risk? Will counselling designed to increase HDL concen- 8 tration rather than specifically to lower LDL 
cholesterol concentration improve our capacity to defer or prevent CHD? Answers as yet we do not have, but we might be a little closer to the truth.

\section{G. J. MILLER}

MRC External Scientific Staff, Caribbean Epidemiology Centre, PO Box 164, Federation Park, Port of Spain, Trinidad, West Indies

\section{References}

Bondjers, G., Olsson, G., Nyman, L. L., and Bjokerud, S. (1977). High density lipoprotein (HDL) dependent elimination of cholesterol from normal arterial tissue in man. Proceedings of the Fourth International Symposium on Atherosclerosis. Edited by G. Schettler, Y. Goto, Y. Hata, and G. Klose, Springer-Verlag, Berlin.

Brown, M. S., and Goldstein, J. L. (1976). Familial hypercholesterolemia: a genetic defect in the lowdensity lipoprotein receptor. New England Journal of Medicine, 294, 1386-1390.

Castelli, W. P., Doyle, J. T., Gordon, T., Hames, C. G., Hjortland, M. C., Hulley, S. B., Kagan, A., and Zukel, W. J. (1977). Alcohol and blood lipids. The cooperative lipoprotein phenotyping study. Lancet, 2, 153-155.

Glomset, J. A. (1970). Physiological role of lecithincholesterol acyltransferase. American Journal of Clinical Nutrition, 23, 1129-1136.
Gofman, J. W., Hanig, M., Jones, H. B., Lauffer, M. A., Lawry, E. Y., Lewis, L. A., Mann, G. V., Moore, F. E., Olmsted, F., Yeager, J. F., Andrus, E. C., Barach, J. H., Beams, J. W., Fertig, J. W., Page, I. H., Shannon, J. A., Stare, F. J., and White, P. D. (1956). Evaluation of serum lipoprotein and cholesterol measurements as predictors of clinical complications of atherosclerosis and longevity. Circulation, 14, 691-741.

Gofman, J. W., Young, W., and Tandy, R. (1966). Ischemic heart disease, atherosclerosis and longevity. Circulation, 34, 679-697.

Gordon, T., Castelli, W. P., Hjortland, M. C., Kannel, W. B., and Dawber, T. R. (1977). High density lipoprotein as a protective factor against coronary heart disease. American Journal of Medicine, 62, 707-714.

Miller, G. J., and Miller, N. E. (1975). Plasma-highdensity-lipoprotein concentration and development of ischaemic heart disease. Lancet, 1, 16-19.

Miller, N. E., Forde, O. H., Thelle, D. S., and Mjos, O. D. (1977). The Tromso heart study. High-density lipoprotein and coronary heart disease: a prospective case-control study. Lancet, 1, 965-968.

Nicholls, A. C., and Thomas, M. (1977). Coxsackie virus infection in acute myocardial infarction. Lancet, 1, 883-884.

Virchow, R. (1856). Gesammelte Abhandlungen zur Wissenschaftlichen Medizin. Medinger, Frankfurtam-Main.

Yano, K., Rhoads, G. G., and Kagan, A. (1977). Coffee, alcohol and risk of coronary heart disease among Japanese men living in Hawaii. New England Journal of Medicine, 297, 405-409. 